\title{
A Patient-Centered Conceptual Model of Symptoms and Their Impact in Early Parkinson's Disease: A Qualitative Study
}

\author{
Hannah Staunton ${ }^{\mathrm{a}, *}$, Kim Kelly ${ }^{\mathrm{b}}$, Louise Newton ${ }^{\mathrm{c}}$, Mathias Leddin ${ }^{\mathrm{d}}$, Raul Rodriguez-Esteban ${ }^{\mathrm{d}}$, \\ K. Ray Chaudhuri ${ }^{\mathrm{e}}$, Daniel Weintraub ${ }^{\mathrm{f}, \mathrm{g}}$, Ronald B. Postuma ${ }^{\mathrm{h}}$ and Pablo Martinez-Martin ${ }^{\mathrm{i}}$ \\ ${ }^{a}$ Roche Products Limited, Welwyn Garden City, UK \\ ${ }^{\mathrm{b}}$ Clinical Outcomes Solutions, Tucson, AZ, USA

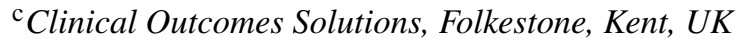 \\ ${ }^{\mathrm{d}}$ Roche Innovation Center Basel, F. Hoffmann-La Roche Ltd, Basel, Switzerland \\ ${ }^{\mathrm{e}}$ Parkinson Foundation Centre Kings College Hospital and Institute of Psychiatry, Psychology and Neuroscience, \\ King's College London, London, UK \\ ${ }^{\mathrm{f}}$ Department of Psychiatry, Perelman School of Medicine at the University of Pennsylvania, Philadelphia, PA, \\ USA \\ garkinson's Disease Research, Education and Clinical Center, Philadelphia Veterans Affairs Medical Center, \\ Philadelphia, PA, USA \\ ${ }^{\mathrm{h}}$ Department of Neurology, Montreal Neurological Institute, Montreal, Canada \\ ${ }^{\mathrm{i}}$ Center for Networked Biomedical Research in Neurodegenerative Diseases (CIBERNED), Carlos III Institute \\ of Health, Madrid, Spain
}

Accepted 21 September 2021

Pre-press 13 October 2021

\begin{abstract}
.
Background: Individuals with Parkinson's disease (PD) develop a significant disease burden over time that contributes to a progressive decline in health-related quality of life. There is a paucity of qualitative research to understand symptoms and impacts in individuals with early-stage PD (i.e., Hoehn and Yahr stage 1-2 and $\leq 2$ years since diagnosis).

Objective: The collection of qualitative data to inform the selection of clinical outcome assessments for clinical trials is advocated by regulators. This patient-centered, multistage study sought to create a conceptual model of symptoms and their impact for individuals with early-stage PD.

Methods: Symptoms and impacts of PD were gathered from a literature review of qualitative research, a quantitative social media listening analysis, and qualitative patient concept elicitation interviews $(n=35)$. Clinical experts provided input to validate and finalize the concepts.

Results: The final conceptual model consisted of 27 symptoms categorized into 'motor' or 'non-motor' domains, and 39 impacts divided into five domains. Most frequently reported symptoms in early-stage PD were 'tremors' (89\%), 'stiffness and rigidity', and 'fatigue' (69\%, both). Most frequently reported impacts included 'anxiety' (74\%), 'eating and drinking' (71\%), followed by 'exercise/sport' and 'relationship with family/family life' (66\%, both).
\end{abstract}

${ }^{*}$ Correspondence to: Hannah Staunton, Roche Products Limited, Hexagon Place, Falcon Way, Shire Park, Welwyn Garden
City, UK, AL7 1TW. Tel.: +44 01707 366726; E-mail: hannah. staunton@roche.com. 
Conclusion: This study provides initial insights relating to the symptom and impact burden of early-stage PD patients. The conceptual model can be used to help researchers to develop and select optimal patient-centered outcomes to measure treatment benefit in clinical trials. These findings could inform future qualitative research and the development of outcomes specifically for early-stage PD patients.

Keywords: Impacts, Parkinson's disease, qualitative research, quality of life, social media, symptoms

\section{INTRODUCTION}

Individuals with Parkinson's disease (PD) experience a significant disease burden over time which can include a range of motor (tremors, stiffness, gait difficulties, and loss of balance) and non-motor symptoms (sleep disorders, fatigue, pain, depression, anxiety, psychosis, cognitive decline, and dysautonomia, among others) [1,2]. As these motor and non-motor symptoms increase, individuals with PD progressively lose their independence and autonomy [3]. This loss has a significant impact on patients' health-related quality of life (HRQoL) and places an increased responsibility on caregivers and family members [4]. The physical and psychosocial burden of PD is relatively well documented in individuals with moderate-to-advanced disease $[5,6]$. Conversely, a paucity of open-ended qualitative literature exists regarding the impact of the earlier stages of PD, with a lack of systematic qualitative studies from a patient-centered perspective. While open-ended interview data are lacking, a recent survey by Port et al. has demonstrated that non-motor symptoms are present from the very earliest and prodromal stages of PD [7]. Moreover, problems with walking, balance and falls, speech problems, freezing of gait, and dyskinesia are relatively mild in early disease, but become increasingly important with disease progression compared with tremor, stiffness, and psychological health, which were found to decrease in importance with disease progression [7].

Within the context of clinical trials for therapies in early-stage PD, it is important to assess symptoms and changes in functional abilities that are relevant and meaningful to such a cohort. Obtaining qualitative insights from patients is recommended in the U.S. Food \& Drug Administration (FDA) Patient-Reported Outcomes guidance [8] and PatientFocused Drug Development guidance series [9] when selecting and developing clinical outcome assessments (COA) for the comprehensive evaluation of treatment efficacy. In addition, understanding the patient perspective regarding relevant symptoms and impacts can be informative in clinical practice to ensure the use of outcomes of importance to patients.
To this end, this patient-centered, multistage study was designed to inform the development of a conceptual model of PD in recently diagnosed individuals (patients within 2 years of diagnosis, Hoehn \& Yahr (H\&Y) stage 1-2). A conceptual model provides a visual depiction of the interrelationships between disease-related symptoms and their impact, as experienced by patients [10]. It is designed to provide a framework from which unmet needs can be easily conveyed and understood. A conceptual model can be used to determine the suitability of an existing outcome assessment by reviewing the extent to which it measures patient-relevant aspects of the disease as captured in the model. This is especially useful in disease areas where outcome assessments exist for similar or overlapping conditions (or in the case of PD for more advanced disease populations) but are potentially absent for the condition of interest. Subsequently, a conceptual model can identify relevant clinical endpoints and ensure that symptoms/impacts considered important to the patient are assessed in future clinical research trials and in clinical practice.

\section{METHODS}

Insights on PD were gathered using a threepronged approach (Fig. 1): 1) a review of published qualitative literature; 2) quantitative social media listening; and 3) concept elicitation (CE) interviews with individuals diagnosed with early-stage PD.

Information from the three sources was gathered to create a preliminary conceptual model. A group of clinical therapeutic area experts (TAEs) provided input throughout the process to validate and finalize the model.

\section{Literature review of qualitative research}

\section{Study design}

The literature search was conducted in OVID using EMBASE, MEDLINE, and PsycINFO databases. A secondary 'grey' literature search (which captured research by organizations outside of the traditional publishing channels) was conducted to ensure that all relevant studies/information were captured in the 

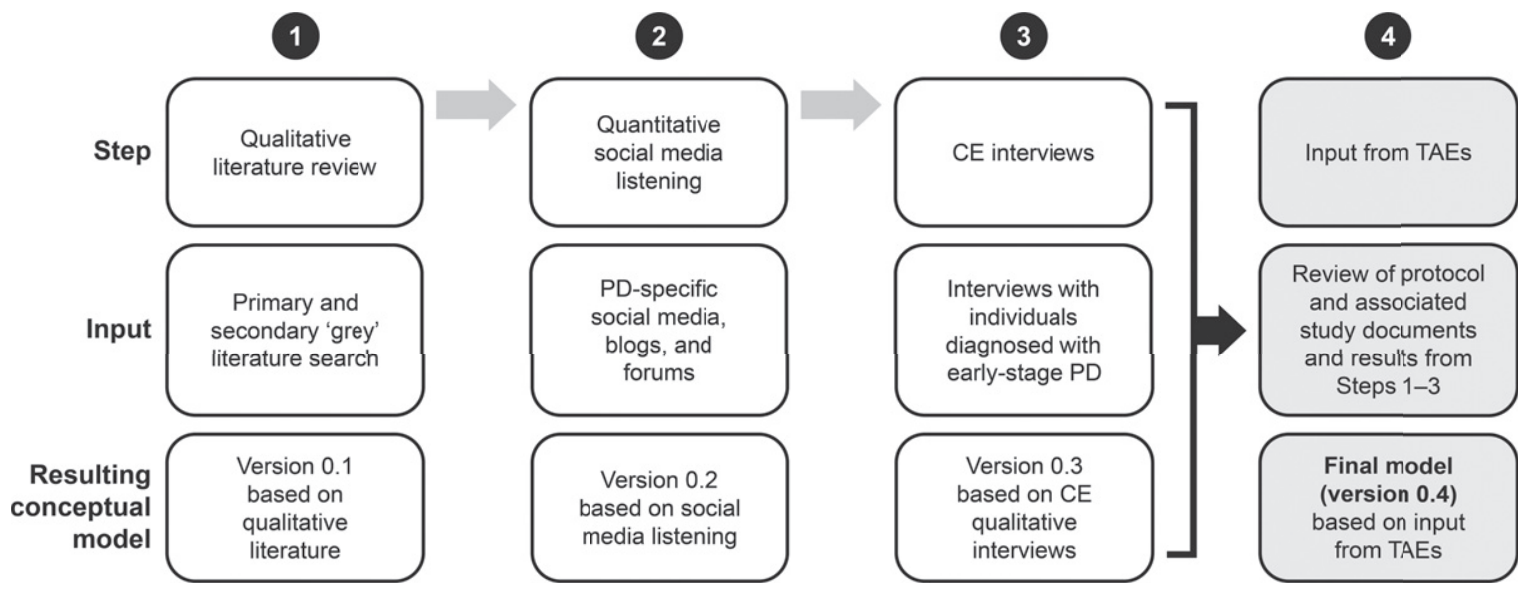

Fig. 1. Development of the PD Conceptual Model.

review. This included a search of conference proceedings and literature from the National Institutes of Health.

\section{Search strategy}

A search strategy of the literature included terms associated with PD, along with qualitative terms (e.g., focus group, interview, narrative, etc.) and symptoms, impacts and HRQoL concepts (e.g., tremor, constipation, daily living, etc.). The search was limited to research articles or reviews published between 2000 and 2017. An article was excluded if a) it was a duplicate; b) it reported on a disease unrelated to PD; c) the study design was not preliminary or descriptive qualitative research, or the primary outcome measure was not evaluated qualitatively; d) the article was published outside of the set dates; or e) it was not written in English.

\section{Data extraction and analysis}

The data were extracted and analyzed by a researcher based on the full assessment criteria including study title; author and year; indication and stage of PD; study design, outcome measure discussed; type of qualitative method; interview guide description/goals; and strengths/limitations of research. A preliminary conceptual model (version 0.1 ) was created which focused on the core symptoms and impacts, raised in $\geq 1 / 3$ of the articles reviewed.

\section{Quantitative social media listening}

\section{Search strategy and data extraction and analysis}

English-speaking PD-specific social media, blogs and forums were identified through a Google online search using the disease name. Text mining of the search results was performed using a tool called Linguamatics I2E to focus on the symptoms and impacts of early-stage PD reported by caregivers and individuals with mention of a self-confirmed diagnosis. The focus was on symptoms and impacts reported to have happened before or around the time of diagnosis. The I2E query strategy examined language patterns that suggested patients describing symptoms or impacts. The results of these queries were manually mapped to expert-chosen categories.

To reduce bias, text mining queries were not restricted to a list of symptoms, but instead used multiple word patterns that could be associated with statements regarding symptoms and impacts [11]. The original language was then analyzed by information scientists. All data were downloaded from publicly available social networking sites and communities with neither access nor mining restrictions.

The preliminary conceptual model was modified (version 0.2) to include additional symptoms and impacts that were raised in the social media listening exercise, that were corroborated by the published literature and clinical experts. The concepts in the preliminary model (version 0.2 ) informed the semistructured $\mathrm{CE}$ interview guide.

\section{Ethical considerations}

This work is compliant with ethical guidelines for the collection and analysis of user-generated content on open internet platforms. Data were downloaded only from open health social networking sites and communities. No information from restricted data areas was downloaded (i.e., content that requires an 
ID or password for access). No aggregation or enrichment of data on an individual was performed.

\section{CE interviews}

\section{Interview design}

CE interviews were conducted to validate the preliminary conceptual model generated from the literature and social media listening analysis. The 1-hour, semi-structured interviews were open-ended by design to encourage participants to spontaneously report on their disease experiences. When needed, more direct questioning was employed by the interviewer to elicit greater detail. Guidance from the FDA relating to the conduct of qualitative interviews using CE methods was followed [12]. This data collection approach generated participant-driven insights and allowed for a bottom-up, thematic analysis of the data to inform changes to the preliminary conceptual model.

\section{Interview population (eligibility criteria)}

The aim was to recruit 20 participants in the US (fluent English speakers), eight participants in France (fluent French speakers), and eight participants in Germany (fluent German speakers) of any race or gender and aged $\geq 40-80$ years who had a clinical diagnosis of early-stage PD ( $\leq 2$ years) and H\&Y stage 1-2. France and Germany were selected as target countries for recruitment to provide insight into potential differences between participants' descriptions and experiences based on a US versus a non-US sample.

Additional eligibility criteria included: a) the presence of idiopathic PD with bradykinesia plus one of the other cardinal signs of PD (resting tremor, rigidity), without any other known or suspected cause of PD [13]; b) individuals that had been diagnosed with $\mathrm{PD} \leq 2$ years at the time of the interview and were untreated or treated with monoamine oxidase $\mathrm{B}$ inhibitors; c) individuals that were classified as stage $1,1.5$, or 2 based on the modified H\&Y scale, or 1 or 2 based on the original H\&Y scale; d) individuals either free of dopaminergic PD medication or who had been taking dopaminergic PD treatment $<6$ months at the time of the interview and; e) individuals who were cognitively and physically capable of participating in an hour-long, face-to-face or telephone interview.

\section{Recruitment}

Target recruitment quotas aimed to ensure that the study population adequately represented the patient population, specifically to obtain a spread of age, education, ethnicity, and PD status. Locally based external agencies recruited participants for the study in the US, Germany, and France. Additional participants were recruited via a direct-to-patient recruitment strategy using the Fox Trial Finder via the Michael J. Fox Foundation (MJFF). Written informed consent was obtained prior to the conduct of any research-related activities.

\section{Interview procedures}

Interviews with individuals lasted approximately 60 minutes. Trained qualitative interviewers used a semi-structured discussion guide to encourage participants to spontaneously discuss concepts. A narrative at the beginning of the interview was designed to orientate the participant towards discussion of the early signs, symptoms and impacts of PD. This was followed by a series of focused questions to explore those symptoms and impacts that the participant spontaneously reported on his/her own. Once the participant noted that he/she had exhausted all of the symptoms and impacts he/she had experienced, the interviewer then went through a list of symptoms and impacts that were identified as commonly experienced by early-stage PD patients in the previously conducted literature review of qualitative research. This approach was undertaken to ensure a systematic approach to discussing symptoms and impacts associated with the disease and is in line with best practices for patient-reported qualitative research [14]. In addition, patients were asked to identify the three 'worst' symptoms and impacts that they associated with PD.

\section{Data analysis}

All interviews were audio recorded and transcribed verbatim by a professional transcription company into a Microsoft ${ }^{\circledR}$ Word document for analysis. NVivo v11.0, a qualitative data analysis software program, was used to assist researchers with the coding and analysis of qualitative data. A thematic analysis was undertaken to code the data from the CE interviews in order to identify and discover any themes or patterns in the data. Symptoms and impacts were coded as 'spontaneous (S)' or 'probed (P)' at the first point of mention during the interview, in order to identify those that were most frequently described by participants without prompting by the interviewer. To support the validity of the conceptual model developed based on the semi-structured qualitative interviews, interview transcripts were analyzed to ensure concept saturation had been met using 
approaches aligned with current scientific guidance (i.e. all significant concepts emerged in the interview sample with no new concepts being introduced in the final set of interviews) $[12,15,16]$. Conceptual saturation based on the US data (three subgroups) was performed and then compared with the European subgroup to explore any key differences at the country level.

\section{Ethical considerations}

The semi-structured interviews were conducted in compliance with Good Clinical Practice guidelines, including International Conference on Harmonisation Guidelines [17] and consistent with the most recent version of the Declaration of Helsinki [18]. In addition, all applicable local laws and regulatory requirements were adhered to throughout the semi-structured interviews. Study documents were approved by the Copernicus Group Independent Review Board in the US (COS1-17-552) and by the Freiburger Ethik-Kommission International in Germany. Ethical approval was not required per the French guidelines at the time of the interviews.

\section{Additional steps}

In order to ensure a robust study design and interview guide, a panel of four clinical TAEs (R.C., D.W., R.P., P.M.M.) reviewed the protocol and associated study documents.

\section{RESULTS}

\section{Literature review of qualitative research}

The literature search resulted in 943 articles. Titles and abstracts were analyzed, and articles were removed based on the inclusion and exclusion criteria (workflow shown in Supplementary Figure 1). A selection of 25 potentially relevant articles underwent a full-text review, with 19 articles determined as appropriate for inclusion. Thirteen core (i.e., mentioned by $1 / 3$ or more of the articles) symptoms and 13 core impacts were extracted from the articles. Core symptoms were categorized into motor or non-motor symptoms and the frequency of their mentions analyzed (Supplementary Figure 2A). 'Tremors' (16/19 articles, 84\%) was the most frequently reported symptom in PD, followed by 'rigidity', 'postural instability' and 'fatigue' (9/19 articles, $47 \%$, for each). The most frequently reported impact of PD was 'relationship with others' (12/19 articles, 63\%), particularly reliance on fam- ily members/caregivers (Supplementary Figure 2B). The reduction and impairment of 'mobility' (12/19 articles, 63\%) was also frequently raised. Following clinical input a preliminary conceptual model (version 0.1) was drafted from the literature review including 15 core symptoms and 12 core impacts associated with PD (Supplementary Figure 3A). The literature review was designed to focus on each of the various stages of PD, including early-stage PD. However, as the majority of articles did not provide information regarding time since PD diagnosis of the patient population studied and information about Hoehn and Yahr stage was not consistently provided it became apparent that early- versus late-stage PD symptoms and impacts could not be easily separated in order to develop a specific conceptual model for each stage. Therefore, although the literature review included symptoms and impacts of relevance in a broader PD population, conducting social media listening research and CE interviews was an important next step to identify concepts that were of specific relevance to early-stage PD patients.

\section{Quantitative social media listening}

Seventeen social media blogs and forums specific to PD (sources provided upon request) were analyzed and a total of 23,756 documents were downloaded. In total, 267 individuals with PD or their caregivers describing disease symptoms and 166 describing impacts were included in this analysis. For privacy reasons, no effort was made to uniquely identify patients across different sites and therefore some patients could be duplicates.

The distribution of symptoms and impacts from the quantitative social media listening was mapped to the preliminary conceptual model generated from the literature review (Fig. 2A and 2B include all symptoms and impacts appearing at a frequency of $\geq 1 \%$ of the overall sample, respectively). Mapping of the concepts revealed considerable overlap, which, in turn, confirmed the validity of the concepts in the preliminary conceptual model based only on the literature review. Symptoms were again categorized as motor or non-motor (Fig. 2A). 'Tremors' $(\mathrm{N}=80,30 \%)$ and 'rigidity' $(\mathrm{N}=35,13 \%)$ were the most frequently mentioned motor symptoms, consistent with the literature review findings. The impact on 'fine motor skills' was the most frequently mentioned impact, reported by $27 \%$ of individuals $(\mathrm{N}=72)$. This was followed by the impacts on 'mobility' $(\mathrm{N}=51,19 \%)$. In the literature review, both of these concepts ("fine 


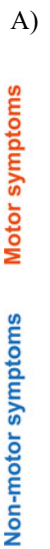
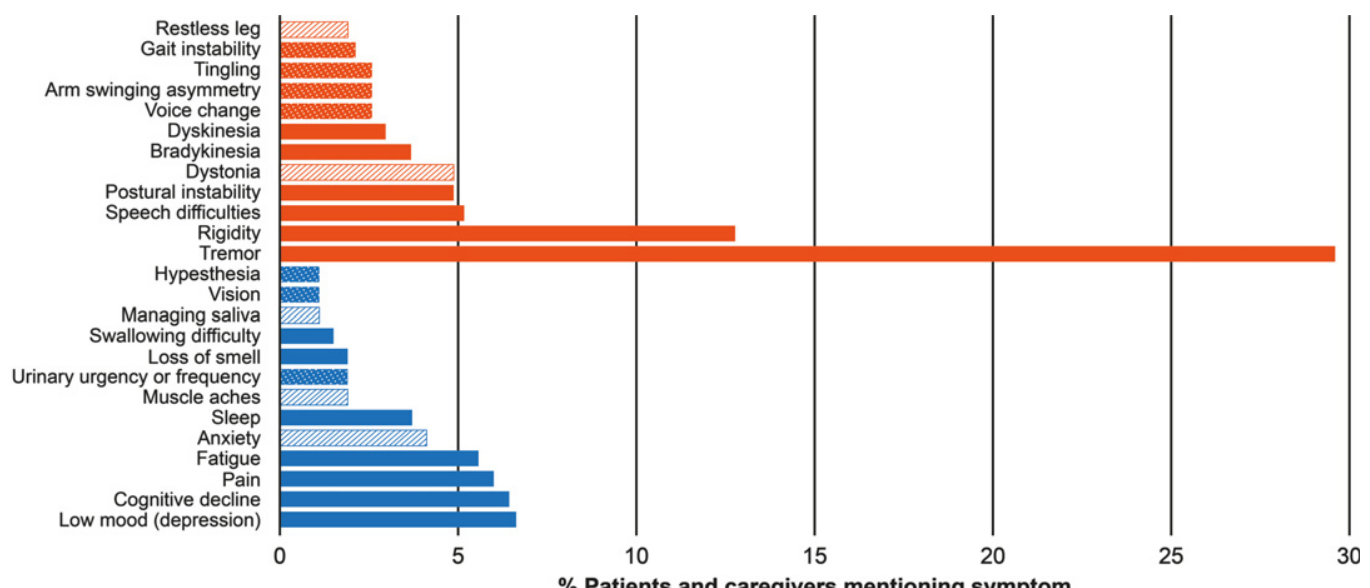

B)

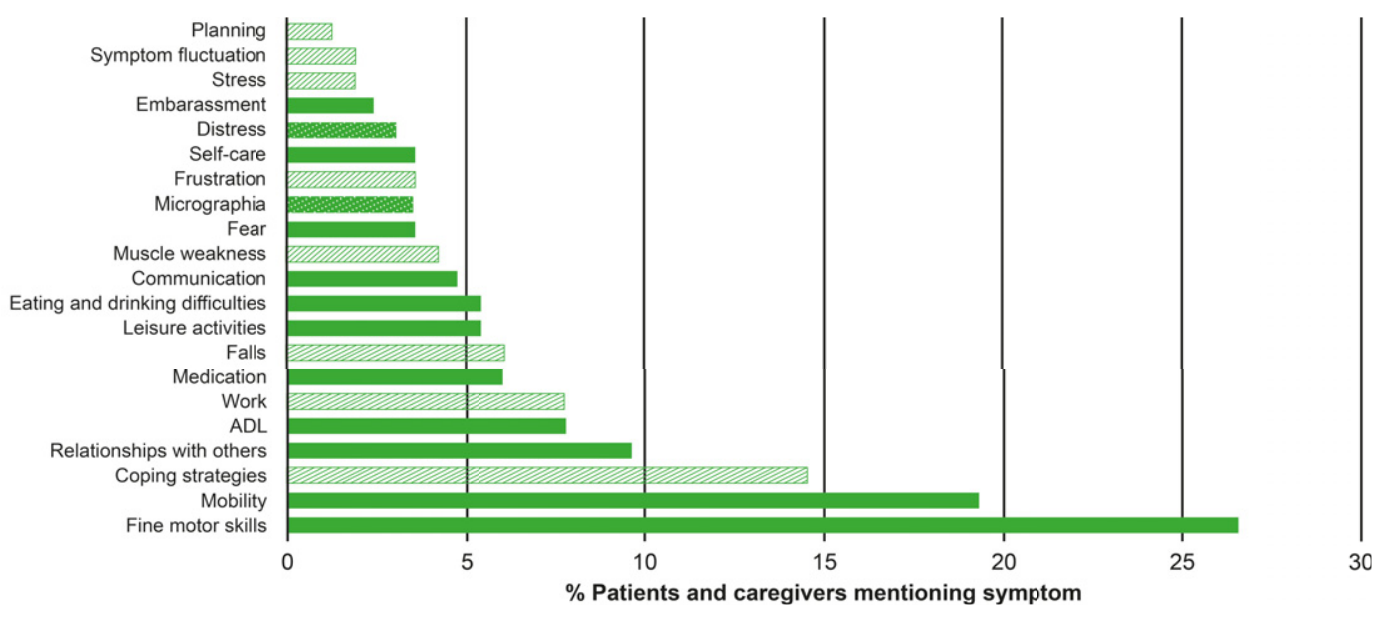

Fig. 2. Distribution of symptoms (A) and impacts (B) mentioned in social media sources* mapped to the concepts identified in the preliminary conceptual model. *Includes all symptoms appearing at a frequency of $\geq 1 \%$ of overall sample. Solid bars indicate symptoms identified in the literature review and already included in the provisional conceptual model (v0.1). Bars with a hashed pattern indicate symptoms identified in the literature review in $<1 / 3$ of reviewed publications but not included in the conceptual model. Bars with a dotted pattern indicate newly identified symptoms in the social media listening which were asked about in the CE interviews. *Includes all impacts appearing at a frequency of $\geq 1 \%$ of overall sample. Solid bars indicate impacts identified in the literature review and already included in the provisional conceptual model (v0.1). Bars with a hashed pattern indicate symptoms identified in the literature review in $<1 / 3$ of reviewed publications but not included in the conceptual model. Bars with a dotted pattern indicate newly identified symptoms which were asked about in the CE interviews. ADL, activities of daily living; CE, concept elicitation.

motor skills' and 'mobility') were raised in $42 \%$ and $63 \%$ of the articles reviewed, respectively (Supplementary Figure 2B).

Based on their frequency of mentions in the social media sources analyzed (approximately $5 \%$ of sample raising concept), alongside support from published literature (i.e., raised in $<1 / 3$ of the literature articles reviewed), the symptoms 'dystonia' and 'anxiety' were added, and the impacts 'work', 'coping strategies' and 'falls' were added to the second iteration of the preliminary conceptual model (version 0.2; Supplementary Figure 3B).
Seven symptoms identified through the social media analysis were not described in the literature, including 'tingling', 'voice changing', 'arm swing asymmetry', 'gait instability' (described by patients as 'vertigo'), 'urinary urgency or frequency', 'vision' and 'hypesthesia'. However, all were infrequently mentioned in the social media listening exercise $(<5 \%)$ and therefore not included in the preliminary conceptual model (version 0.2). In addition, two impacts, 'micrographia' and 'distress', were newly identified, also with low frequency $(<5 \%)$ and therefore not included in the preliminary conceptual 
model (version 0.2). Finally, after seeking expert clinical opinion, additional symptoms and impacts were also incorporated into to the preliminary conceptual model (version 0.2) including 'constipation', 'loss of smell', 'daytime sleepiness', 'sleep problems', 'social isolation' and 'communication'.

Thus, the preliminary conceptual model version 0.2 developed for testing in the $\mathrm{CE}$ interviews included 18 core motor and non-motor symptoms and 16 core impacts associated with PD based on findings from the literature review and social media listening research. The concepts included in the preliminary model were also added to the $\mathrm{CE}$ interview guide for further exploration.

\section{Qualitative CE interviews}

The demographics and clinical characteristics of interview participants are reported in Table 1. The sample consisted of 35 participants (37\% female; $63 \%$ male) who were diagnosed for an average of 10.8 months (range 3-23 months) at the time of interview. The participants were predominantly from the US $(n=19)$ and Germany $(n=15)$, with only one French patient. The mean age was 65.1 years, with over half of the sample being retired. Five of the 35 participants were reported as needing a full-time caregiver. Most participants $(69 \%)$ were in $\mathrm{H} \& \mathrm{Y}$ stage 1 and reported being either in 'very good' or 'good' health $(77 \%$, combined). Of the remaining participants, $20 \%$ were H\&Y stage 2. Most participants (60\%) interviewed were untreated for PD.

Following the CE interviews, the preliminary conceptual model was revised to reflect the symptoms and impacts reported by participants (version 0.3; Supplementary Figure 3C). A concept was included in the conceptual model if it had at least four mentions by different participants (i.e., $>10 \%$ of the sample endorsed). In total, 26 symptoms were discussed by $>10 \%$ of the sample and divided into 'motor' or 'non-motor'. Thirty-nine impacts were discussed by $>10 \%$ of the sample and divided into five domains: emotional/psychological functioning; social role functioning; activities of daily living (ADL); physical functioning; and fine motor skills. Findings from the CE interviews are discussed below.

The symptom 'tremors' was reported (either spontaneously or in response to probing by the interviewer) by nearly all participants $(\mathrm{N}=31 / 35,89 \%)$, and was the most frequently reported symptom, consistent with both the literature review and quantitative social media listening findings. 'Tremors' were described as shivering, shaking, and twitching, often localized to one side of the body or limb. Participants also reported 'stiffness and rigidity' and 'fatigue' as frequent symptoms $(\mathrm{N}=24 / 35,69 \%$, both). 'Stiffness and rigidity' were described as tightness, a clenching of the muscles, contracted or stiff muscles. 'Fatigue' $(\mathrm{N}=24 / 35,69 \%)$ was generally described as a physical symptom. When participants were asked to identify the 'worst' symptoms of PD, participants most frequently identified 'tremors' $(\mathrm{N}=19 / 35$, $54 \%)$, 'stiffness and rigidity' $(\mathrm{N}=8 / 35,23 \%)$ and 'fatigue' $(\mathrm{N}=7 / 35,20 \%)$, consistent with the most frequent symptoms.

Overall, 'anxiety/worry/fear' was the most frequently reported impact by participants $(\mathrm{N}=28 / 35$, $80 \%)$, followed by 'eating and drinking' $(\mathrm{N}=25 / 35$, $71 \%)$, 'annoyance or bother' $(\mathrm{N}=24 / 35,69 \%)$, 'exercise/sport' and 'relationship with family/family life' ( $\mathrm{N}=23 / 35,66 \%$ respectively). Participants were asked to report which three impacts were the 'worst'; those most frequently reported consisted of 'anxiety/worry/fear' ( $\mathrm{N}=8 / 35,23 \%)$, followed closely by 'impact on walking' $(\mathrm{N}=6 / 35,17 \%)$. 'Eating and drinking', 'travel and driving' and 'handwriting' were all equally reported $(\mathrm{N}=3 / 35$, 9\%). Representative quotes from participants on these reported symptoms and impacts are provided in Fig. 3A and 3B, respectively.

Overall symptom and impact concepts were well represented across both the US and European subgroups. Certain concepts related to social and emotional functioning differed at a country level. Participants in Europe $(n=16)$, compared with their US counterparts $(n=19)$, stated that they were more likely to deny, downplay or ignore their illness (63\%, Europe; $11 \%$, US), and reported increased social isolation $(56 \%$, Europe; $11 \%$, US) and stigma (50\%, Europe; $16 \%$, US). These concepts may be linked as greater stigma may lead patients to be more likely to deny, downplay or ignore their illness or socially isolate.

Saturation analysis revealed that in the US sample, 22/26 symptoms were raised in the first two sets of interviews. One symptom, 'insomnia', failed to reach saturation within the US population, as it was only identified in the last set and by one participant within this set. However, insomnia was raised in the European sample. Two symptoms (involuntary movements and loss of physical sensation) were only reported by US participants when probed and one concept (freezing of gait) was not raised in any of the three sets of US interviews but was raised in 
Table 1

CE Qualitative interviews - patient demographics and clinical characteristics

\begin{tabular}{|c|c|c|c|}
\hline & $\begin{array}{c}\text { Total } \\
(\mathrm{N}=35)\end{array}$ & $\begin{array}{l}\text { United States } \\
\quad(n=19)\end{array}$ & $\begin{array}{l}\text { Europe } \\
(n=16)\end{array}$ \\
\hline \multicolumn{4}{|l|}{ Patient-reported demographic, n (\%) } \\
\hline \multicolumn{4}{|l|}{ Sex } \\
\hline Female & $13(37.1)$ & $9(47.4)$ & $4(25.0)$ \\
\hline Male & $22(62.9)$ & $10(52.6)$ & $12(75.0)$ \\
\hline \multicolumn{4}{|l|}{ Age } \\
\hline $\mathrm{N}$ & 31 & 15 & 16 \\
\hline Mean (SD) & $65.1(9.47)$ & $68.2(10.19)$ & $62.1(7.97)$ \\
\hline \multicolumn{4}{|l|}{ Race* } \\
\hline White/Caucasian & $18(51.4)$ & $18(94.7)$ & 0 \\
\hline Missing & $17(48.6)$ & $1(5.3)$ & $16(100)$ \\
\hline \multicolumn{4}{|l|}{ Ethnicity } \\
\hline Non-Hispanic/Latino & $18(51.4)$ & $18(94.7)$ & 0 \\
\hline Missing & $17(48.6)$ & $1(5.3)$ & $16(100)$ \\
\hline \multicolumn{4}{|l|}{ Work status } \\
\hline Employed (full/part time) & $10(28.6)$ & $4(21.1)$ & $6(37.5)$ \\
\hline Retired & $21(60.0)$ & $12(63.2)$ & $9(56.3)$ \\
\hline Other/Missing & $4(11.4 \%)$ & $3(15.8 \%)$ & $1(6.3 \%)$ \\
\hline \multicolumn{4}{|c|}{ (Self-reported) limitations in activities of daily living } \\
\hline Not at all & $9(25.7)$ & $7(36.8)$ & $2(12.5)$ \\
\hline Slightly & $22(62.9)$ & $10(52.6)$ & $12(75.0)$ \\
\hline Moderately & $2(5.7)$ & $1(5.3)$ & $1(6.3)$ \\
\hline Totally & $1(2.9)$ & $0(0.0)$ & $1(6.3)$ \\
\hline Missing & $1(2.9)$ & $1(5.3)$ & $0(0.0)$ \\
\hline \multicolumn{4}{|l|}{ Full-time caregiver } \\
\hline Yes & $5(14.3)$ & $3(15.8)$ & $2(12.5)$ \\
\hline \multicolumn{4}{|l|}{ Clinician-reported clinical information } \\
\hline \multicolumn{4}{|l|}{ Months since diagnosis } \\
\hline $\mathrm{N}$ & 31 & 15 & 16 \\
\hline Mean (SD) & $10.8(5.30)$ & $10.0(5.45)$ & $11.6(5.20)$ \\
\hline \multicolumn{4}{|l|}{ H\&Y stage } \\
\hline Stage 1: Unilateral involvement ${ }^{\dagger}$ & $24(68.6)$ & $10(52.6)$ & $14(87.5)$ \\
\hline Stage 2: Bilateral/Midline involvement & $7(20.0)$ & $5(26.3)$ & $2(12.5)$ \\
\hline Missing & $4(11.4)$ & $4(21.1)$ & 0 \\
\hline \multicolumn{4}{|l|}{ Overall health } \\
\hline Very good & $11(31.4)$ & $9(47.4)$ & $2(12.5)$ \\
\hline Good & $16(45.7)$ & $5(26.3)$ & $11(68.8)$ \\
\hline Fair & $4(11.4)$ & $1(5.3)$ & $3(18.8)$ \\
\hline Missing & $4(11.4)$ & $4(21.1)$ & 0 \\
\hline \multicolumn{4}{|l|}{ Treatment } \\
\hline None & $21(60.0)$ & $9(47.4)$ & $12(75.0)$ \\
\hline Botox $^{\ddagger}$ & $1(2.9)$ & $1(5.3)$ & $0(0.0)$ \\
\hline Physical therapy & $3(8.6)$ & $1(5.3)$ & $2(12.5)$ \\
\hline Rasagiline & $4(11.4)$ & $4(21.1)$ & 0 \\
\hline Missing $\S$ & $6(17.1)$ & $4(21.1)$ & $2(12.5)$ \\
\hline
\end{tabular}

* Race and ethnicity questions not included in European forms, thus total $\mathrm{N}=19 .{ }^{\dagger}$ Participants classified as Stage 1.5 on the modified H\&Y scale were considered Stage $1 .{ }^{\ddagger}$ Botox refers to treatment with Botox for foot dystonia. ${ }^{\S}$ Four US Case Report Forms were unable to be obtained and two European Case Report Forms contained incomplete data. CE, concept elicitation; H\&Y, Hoehn \& Yahr; SD, standard deviation.

the European interviews. For impacts 38/39 concepts were raised in the first two sets of US interviews. One concept (stress) was not spontaneously raised in any of the three sets of US interviews but was raised by one participant in the US when probed and in the European sample. In the European sample, 21/26 symptom concepts and 38/39 impact concepts were spontaneously reported by at least one participant. The five symptoms not spontaneously reported in the European subgroup were 'excess saliva/drooling', 'constipation', 'difficulty swallowing', 'urinary dysfunction' and 'speech and language difficulties' and the one impact not mentioned was 'having to plan around PD'. 
A) Patient-reported symptoms quotes

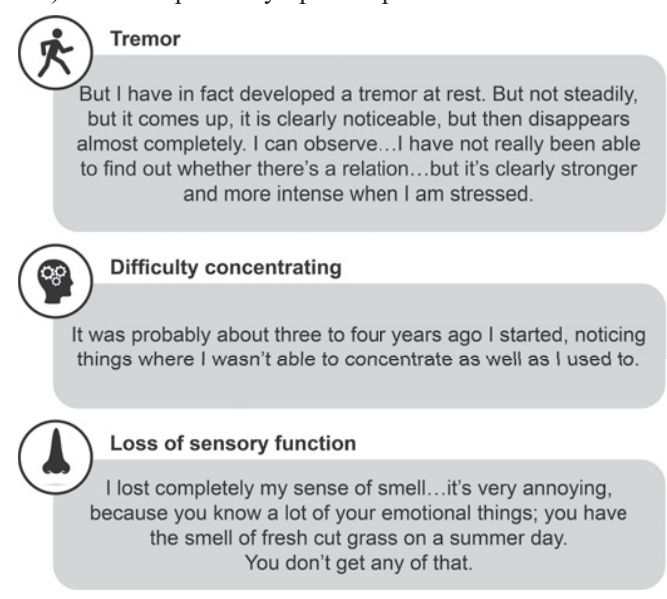

B) Patient-reported impacts quotes

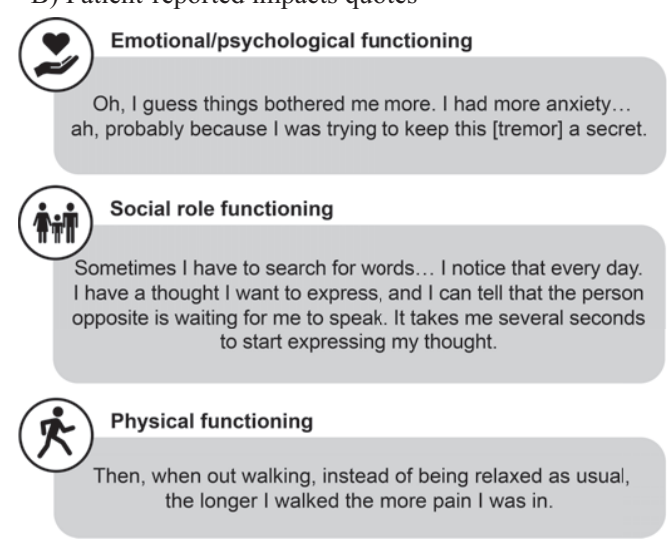

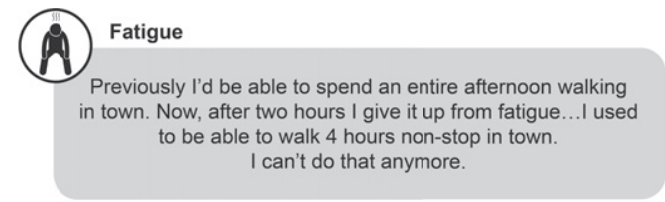

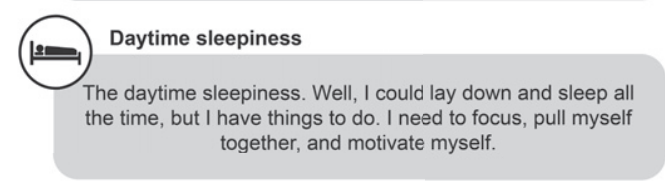

Speech difficulties

Speaking was getting more and more difficult over the past few years... My close friends/family said they didn't, don't, notice anything odd about my speech. But to me it felt really slow and I wasn't speaking fluently, and I was having to take a lot more pauses - it didn't, feel normal to me.

Fig. 3. Patient-reported symptom (A) and impact (B) quotes from the concept elicitation interviews.

\section{Development of a final conceptual model of early-stage $P D$}

Based on the CE interviews, the conceptual model (version 0.3) included 26 symptoms and 39 impacts associated with early-stage PD. Overlap of the symptoms and impacts was observed with the preliminary conceptual model (version 0.2 based on the literature and social media); however, a number of new symptoms $(n=11)$ and impacts $(n=28)$ were identified and other concepts were reworded or relocated in the model through the early-stage PD CE interviews. Following the $\mathrm{CE}$ analysis, version 0.3 of the conceptual model was further refined following feedback and suggestions from clinical TAEs (version 0.4; Fig. 4). Further refinement of the model following expert review ensured that concepts were both patient-centric and clinically valid. Feedback ranged from changes to wording to aid clarity, clarification of the meaning of certain concepts to reconceptualizing and recategorizing others, to more accurately capture the experience of early-stage PD (Supplementary Table 1).

For the symptoms, the main changes included moving a number of concepts previously in the non-motor domain, including 'excessive saliva/drooling', 'change in voice', 'postural issues' and 'speech difficulties', to the motor domain to reflect their clinical manifestation in early-stage PD. In addition, the speech domain was removed, the concept 'language difficulties' was added to the cognitive domain, and 'sleep disturbances' was renamed 'sleep behavior disorders'. Two symptom concepts, 'freezing of gait' and 'uncontrollable muscle contractions', were removed from the final conceptual model based on clinical expert input which suggested a lack of 


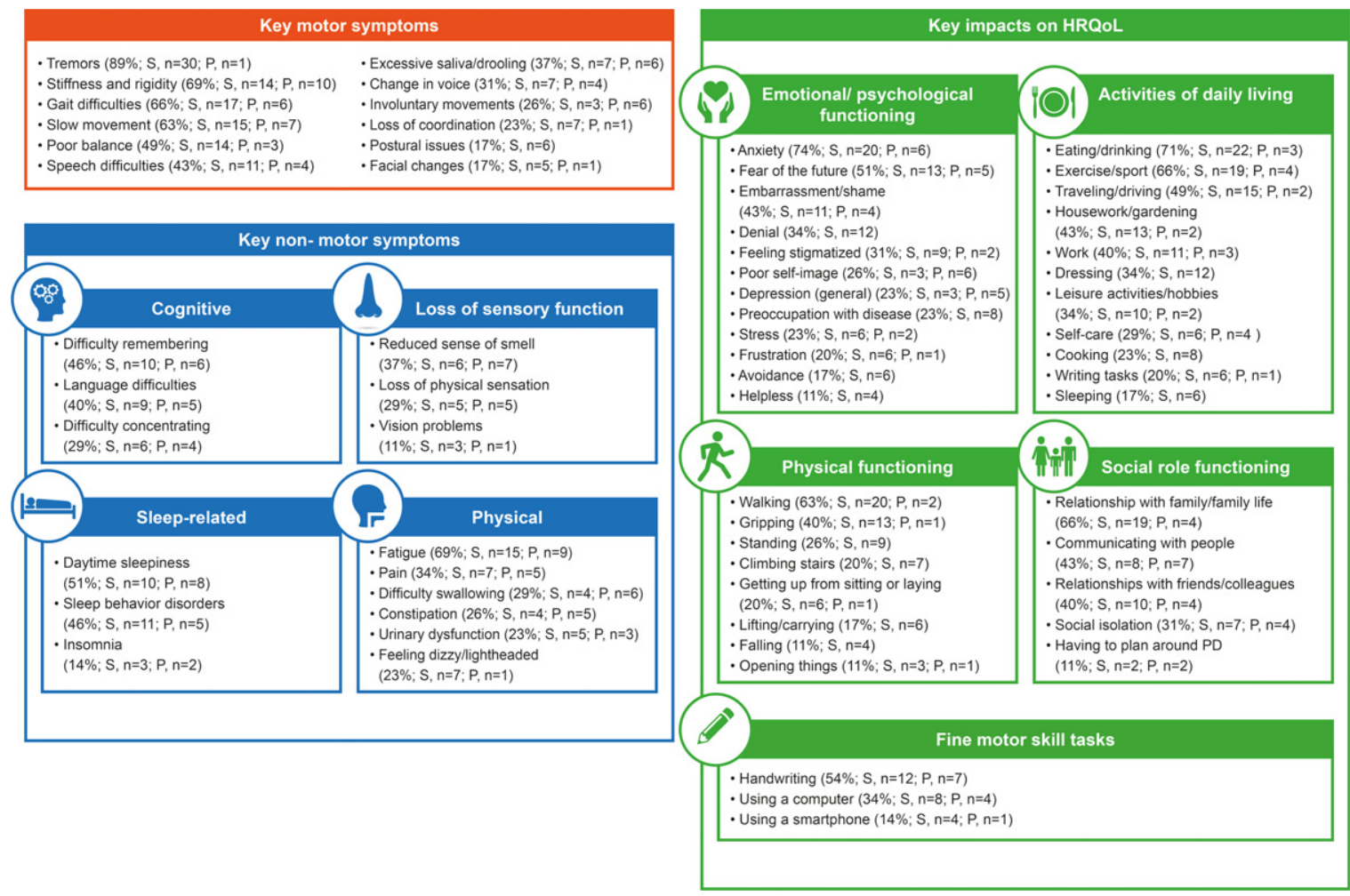

Fig. 4. Final conceptual model. The percentage of interviewees reporting each symptom and impact is shown in parentheses. HRQoL, health-related quality of life; P, probed by researcher; PD, Parkinson's disease; S, spontaneously reported by the patient.

relevance to early-stage PD, and in the case of 'uncontrollable muscle contractions', there was an overlap with 'rigidity' and/or 'involuntary movement'.

With regards to modifications of the impacts, the frequently reported impacts in the 'anxiety/fear' category were modified and split into two separate concepts: 'anxiety' and 'fear of the future'. This allowed for the separation of generalized anxiety (i.e., the general psychological state of the individual) and the fear of the future related to a diagnosis of a progressive, neurodegenerative condition (which does not necessarily reflect a pathologic anxiety state). For impacts, 'annoyance or bother' was removed on the basis that the majority of mentions of feeling annoyed or bothered were in the context of other highly related impacts. All other impacts remained the same based on patients' reports.

Thus, the final conceptual model includes 27 core symptoms (structured into motor and non-motor domains) and 39 core impacts (structured into five domains) associated with PD based on findings from the CE interviews and input from clinical therapeutic area experts, as well as the literature review and social media listening research.

\section{DISCUSSION}

A patient-centered conceptual model of recently diagnosed PD patients was developed based on a literature review of qualitative research, an analysis of social media sources, 35 interviews conducted primarily with individuals with untreated early-stage PD (predominantly from the US and Germany) and clinical expert input. Such qualitative information, as recommended in the FDA PatientReported Outcomes guidance and Patient-Focused Drug Development guidance, is critical for the development of COAs for the comprehensive evaluation of treatment efficacy $[12,19]$. As described by Donatti et al. [10], conceptual models should ideally be developed early in drug development (Phase 1 and 2) to facilitate the selection of COAs as endpoints to support the benefit-risk profile of a treatment. In the case of early-stage PD, COAs should focus on both the motor and non-motor symptom experience as well as the broad array of impacts represented. This conceptual model can be used to map to existing COAs to assess their adequacy in covering the concepts of importance to patients with early-stage PD for use in 
clinical trials or as outcomes in clinical practice. In addition, the conceptual model can be a useful foundation for the development of new COAs in order to comprehensively measure the early-stage PD symptom or impact experience.

The most frequently reported symptom in this study was tremors; this finding complements a recent survey by Port et al. of patients with a diagnosis of PD $<2$ years [7], which found that tremor was the most frequently reported symptom that respondents wished to see an improvement in. Similarly, a cross-sectional analysis of individuals with PD found tremors to be the most bothersome symptom in early $\mathrm{PD}$ ( $\leq 3$ years diagnosis, $n=12,040$ ), raised by $56 \%$ of the sample [20,21]. The next two most frequently reported symptoms (stiffness/rigidity and fatigue) and the most frequently reported impact (anxiety) in this study were also among the 10 most frequently reported symptoms that respondents with a diagnosis of $\mathrm{PD}<2$ years wished to see an improvement in [7]. The additional symptoms reported in Port et al. as most important to improve included dexterity and coordination, movement, cognitive function, slowness, walking, pain and unpleasant sensations, all of which were raised in this study as being relevant in early-stage disease. This consistency in the findings is important given the differences in methodologies between the studies (i.e., indepth interviews versus a survey and cross-sectional analyses).

The conceptual model depicts the heterogeneous and broad nature of the symptom and impact experience in this population. By layering concepts from each methodology, this study provides a comprehensive inventory of early-stage symptoms and brings forth insights into their impact as experienced by individuals with $\mathrm{PD}$ in the years immediately following diagnosis. The first iterations of the model (version 0.1 and version 0.2 ) were based on PD symptoms and impacts. The CE interviews and subsequent expert input were required to ensure specificity of the model to early-stage PD.

The novel approach of including a social media listening analysis resulted in the development of a model that incorporates a uniquely patient-centered perspective; this led to the identification of previously under-reported impacts, such as 'anxiety' and 'fear'. Insights gained were also found to be complementary to those obtained from the qualitative interview study; where both anxiety and fear were raised by participants primarily spontaneously, but also in some instances when probed by the inter- viewer. The benefits of this approach were confirmed by a previous study that also used social media to identify novel aspects of a condition that had not otherwise been reported [22]. In addition, qualitative interviews remain a critical part of understanding the patient's experience of complex diseases such as $\mathrm{PD}$, as in the case of this research, the interviewer could probe and gain additional depth of insights relating to symptoms, impacts, and the lived experience of early-stage PD specifically. While efforts were made to identify a recently diagnosed population in the social media listening exercise, due to the inability to confirm diagnosis, the qualitative interviews provided a needed source of information to understand this early-stage PD population of interest. Interview data were subjected to concept saturation analysis (i.e., the point at which no new concepts emerge) to ensure that all concepts were accurately captured within the final model; this process revealed that both symptom and impact concepts were well represented across the US and European subgroups. Two concepts appeared to be relevant in only one subgroup: 'freezing of gait' was only reported by European participants and 'having to plan around PD' was only reported by US participants. However, when taking into account probed reporting, all other concepts were endorsed by participants in both subgroups. Finally, the involvement of clinical experts in the development of this conceptual model is consistent with previous research and underlines the importance of ensuring a patient-centered as well as clinically accurate depiction of the disease experience [23].

The subtle/mild nature of many of the symptoms and impacts experienced at the early-stage of the disease (e.g., difficulties in holding a drink due to tremors, as opposed to a major limitation of consuming the drink due to difficulties swallowing) became apparent during this study. In addition, several individuals were made aware of symptoms such as moving slowly, altered speech/voice and changes to facial expressions by a caregiver/family member due to the observable nature of these concepts. This may indicate that, for certain symptoms of PD, patient self-reported assessment alone may not be sufficient to detect small changes in symptoms and impacts, and that additional observer-reported assessments completed by caregivers/family members may be of benefit. This is supportive of previous observations; for example, sleep dysfunction such as REM sleep behavior disorder and somnolence are often more accurately reported through a spouse/partner, fam- 
ily or friends [24]. These observations also highlight the important role that friends and family can play, as individuals with PD are more likely to discuss symptoms with close companions before engaging with a healthcare professional [25]. A combination of self-reported and observer-reported assessments focused on subtle changes in functioning that matter to patients at this early-stage of the disease may be required to capture all concepts of relevance, many of which are best known to patients while some concepts are best observed by others (e.g., sleep disturbances, change in facial expression, etc.).

As expected in a conceptual model cataloging early-stage PD symptoms and impacts, cognitive decline was not explicitly reported by interview participants. Some subtle impacts alluding to cognitive decline were identified (e.g., 'communicating with people' or 'word-finding problems within a social setting'), highlighting that impairment of cognitive ability has a potential impact in early disease stages. Cognitive decline or impairment has previously been reported in newly diagnosed PD patients [26], and as a priority to improve for patients with a diagnosis of PD $<2$ years [7]. Further cognitive impacts may perhaps have been elicited if the caregiver perspective was included in conjunction with a cognitive screening battery, such as the Montreal Cognitive Assessment, which has been found to be sensitive to early cognitive decline [27]. Future interview studies from caregivers and physicians could help provide a broader picture of symptoms and impacts in recently diagnosed individuals.

One important finding from this study is that existing COAs, which measure PD clinical symptoms and impacts through standard patient-reported outcomes only, capture broad concepts and may not be sufficiently granular to capture small or subtle changes in functioning. For example, the Parkinson's Disease Quality of Life measure (PDQ-39) and MDS-Unified Parkinson's Disease Rating Scale assess concepts such as 'difficulty holding eating utensils' and 'holding a drink' (PDQ-39 only). Concepts such as 'opening a jar or bottle' and 'typing or using a mouse', which were raised in the interviews as being difficult, are absent from these ADL measures $[28,29]$. A number of therapies currently in development focus on neuroprotection early in the disease process [30]; the ability to better assess the subtle changes that occur in this stage of disease development will be crucial in assessing the efficacy of these emerging treatments.

\section{Limitations of study}

This conceptual model is based on cross-sectional data from patients classified as stage $1,1.5$, or 2 based on the modified H\&Y scale, and thus may not be exhaustive of all symptoms and impacts that could be experienced by patients with early-stage PD, but rather represents initial insights from the patient and expert perspectives.

When considering the different methods employed in this study, the limitations of the literature review included the lack of data available on patient's PD disease duration, illustrating the need for additional exploration of symptoms and impacts in early-stage PD as was conducted in the subsequent parts of this study. The social media analysis limitations included the inability to confirm the diagnosis of the patients and that the sources were not assessed for duplicate patients or caregivers and therefore it is possible that the same patients and caregivers contributed to multiple sources.

With respect to the CE interview stage, the findings included insights from $\mathrm{N}=35$ patients, and represent initial insights that could inform further qualitative research. Regarding the sample included in the $\mathrm{CE}$ interviews, the individuals included were predominantly from the MJFF trial registry and thus may not be representative of the general PD population. In addition, the majority of the participants had not yet received any form of symptomatic treatment, and results may not apply to those already on treatment. Although there may be subtle, important differences between treated and untreated populations, there is some evidence that certain symptoms such as urinary dysfunction, depression, constipation, and orthostatic hypotension can emerge due to levodopa-resistance particularly in advanced stages of the disease [31]. Furthermore, there was a lack of diversity among the interview participants; all US-based participants identified as White/Caucasian (race/ethnicity was not collected in the European centers). Cultural and social influences on the impacts of PD, which may vary between countries, were not considered in this study. Standard of care of early-stage PD also differed between countries. For instance, in France, national guidelines recommend that younger patients $(<60-65$ years old) start with dopamine agonists or monoamine oxidase B inhibitors while individuals aged $>60-65$ years old are prescribed levodopa plus dopa decarboxylase inhibitor [32]. Therefore, a more in-depth comparison of recently diagnosed PD individuals could not be conducted across the 
three countries in the study. Another limitation was that most participants in Europe were male. Lastly, early-stage PD symptoms and impacts significant to a younger PD population may not have been fully captured, as all but one study participant was $>50$ years old. Further studies should be conducted in additional countries to ascertain the generalizability of these findings and to analyze the influence of age, gender, race and ethnicity on symptoms and impacts.

\section{CONCLUSIONS}

Qualitative research with individuals and caregivers is crucial to advancing our understanding of the experience of living with early-stage PD. From this study, it is clear that the impact of symptoms on HRQoL is significant but potentially subtle in individuals with a recent diagnosis of PD. This finding reinforces the need for the development of accurate and reliable patient-centered clinically valid assessments that can detect changes over time in interventional settings. The final conceptual model of early-stage PD which outlines the symptoms and impacts of relevance in this population will help researchers to select optimal patient-centered COAs and develop new outcomes where gaps exist (e.g., the measurement of subtle changes in ADLs), in order to measure treatment benefit in clinical trials enrolling patients with early-stage PD, H\&Y stage 1-2 or for use in clinical practice to ensure the measurement of outcomes of relevance to patients.

\section{ACKNOWLEDGMENTS}

The authors would like to thank all the individuals and their family members/caregivers who participated in this study and Tara Symonds from Clinical Outcomes Solutions for her contributions to the study. Medical writing support was provided by Drs Amanda Gallagher and Michelle Kim at MediTech Media.

This study was funded by F. Hoffmann-La Roche Ltd.

\section{CONFLICT OF INTEREST}

HS is an employee and shareholder of Roche Products Ltd. ML and RRE are employees of F. Hoffmann-La Roche Ltd. LN is an employee of Clinical Outcomes Solutions who are a research consultancy organization paid by Roche Products Ltd to undertake aspects of the research discussed in this manuscript. LN has no conflicts of interest to report.
KK was a former employee of Clinical Outcomes Solution at the time of this research and is currently affiliated with IQVIA Scientific Services, New York NY. KK has no conflicts of interest to report.

KRC, DW, RP, and PMM have received consultancy fees from Roche Products Ltd for this work.

KRC has served on the advisory boards for AbbVie, UCB Inc, Global Kinetics Corporation (GKC), Bial, Cynapsus, Novartis, Lobsor, Stada, Medtronic, Zambon, Profile, Sunovion, Roche, Theravance, Scion, Britannia. Honoraria for lectures have been received from AbbVie, Britannia Pharmaceuticals, UCB, Mundipharma, Zambon, Novartis, and Boehringer Ingelheim. KRC has also received investigator-initiated grants from Britannia Pharmaceuticals, AbbVie, UCB Inc, GKC, Bial, Academic grants: EU, IMI EU, Horizon 2020, Parkinson's UK, National Institutes of Health Research, PDNMG, EU (Horizon 2020), Kirby Laing Foundation, NPF, MRC, and Wellcome Trust.

DW has received research funding or support from Michael J. Fox Foundation for Parkinson's Research, Alzheimer's Therapeutic Research Initiative (ATRI), Alzheimer's Disease Cooperative Study (ADCS), the International Parkinson and Movement Disorder Society (IPMDS), and National Institute on Aging (NIA); honoraria for consultancy from Acadia, CHDI Foundation, Clintrex LLC (Aptinyx, Avanir, Otsuka), Eisai, Janssen, Sage, Signant Health and Sunovion; and license fee payments from the University of Pennsylvania for the QUIP and QUIP-RS.

RP has received grants and personal fees from Fonds de la Recherche en Sante, the Canadian Institute of Health Research, Parkinson Canada, the Weston-Garfield Foundation, the Michael J. Fox Foundation, the Webster Foundation, and personal fees from Takeda, Roche/Prothena, Teva Neurosciences, Novartis, Biogen, Boehringer Ingelheim, Theranexus, GE HealthCare, Jazz Pharmaceuticals, AbbVie, Janssen, CuraSen and Otsuka.

PMM has received honoraria from National School of Public Health (ISCIII), Editorial Viguera and Takeda Pharmaceuticals for lecturing in courses; and from the International Parkinson and Movement Disorder Society (IPMDS) for management of the Program on Rating Scales.

\section{SUPPLEMENTARY MATERIAL}

The supplementary material is available in the electronic version of this article: https://dx.doi.org/ 10.3233/JPD-202457. 


\section{REFERENCES}

[1] Poewe W (2008) Non-motor symptoms in Parkinson's disease. Eur J Neurol 15 Suppl 1, 14-20.

[2] Moustafa AA, Chakravarthy S, Phillips JR, Gupta A, Keri S, Polner B, Frank MJ, Jahanshahi M (2016) Motor symptoms in Parkinson's disease: A unified framework. Neurosci Biobehav Rev 68, 727-740.

[3] Xia R, Mao Z-H (2012) Progression of motor symptoms in Parkinson's disease. Neurosci Bull 28, 39-48.

[4] Martinez-Martin P, Rodriguez-Blazquez C, Forjaz MJ (2012) Quality of life and burden in caregivers for patients with Parkinson's disease: Concepts, assessment and related factors. Expert Rev Pharmacoecon Outcomes Res 12, 221-230.

[5] Deal LS, Flood E, Myers DE, Devine J, Gray DL (2019) The Parkinson's Disease Activities of Daily Living, Interference, and Dependence Instrument. Mov Disord Clin Pract 6, 678-686.

[6] Hurt CS, Weinman J, Lee R, Brown RG (2012) The relationship of depression and disease stage to patient perceptions of Parkinson's disease. J Health Psychol 17, 1076-1088.

[7] Port RJ, Rumsby M, Brown G, Harrison IF, Amjad A, Bale CJ (2021) People with Parkinson's disease: What symptoms do they most want to improve and how does this change with disease duration? J Parkinsons Dis 11, 715-724.

[8] Food and Drug Administration, Guidance for Industry Patient-Reported Outcome Measures: Use in Medical Product Development to Support Labeling Claims, https://www. fda.gov/media/77832/download, Accessed October 23.

[9] Food and Drug Administration, FDA Patient-Focused Drug Development Guidance Series for Enhancing the Incorporation of the Patient's Voice in Medical Product Development and Regulatory Decision Making, https://www.fda.gov/ drugs/development-approval-process-drugs/fda-patientfocused-drug-development-guidance-series-enhancingincorporation-patients-voice-medical, Accessed October 23.

[10] Donatti C, Wild D, Hareendran A (2008) The use of conceptual models, conceptual frameworks, and endpoint models to support label claims of treatment benefit using patient reported outcomes. ISPOR Connect 14, 9-12.

[11] Milward D, Bjäreland M, Hayes W, Maxwell M, Oberg L, Tilford N, Thomas J, Hale R, Knight S, Barnes J (2005) Ontology-based interactive information extraction from scientific abstracts. Comp Funct Genomics 6, 67-71.

[12] Food and Drug Administration, Methods to Identify What is Important to Patients \& Select, Develop or Modify FitFor-Purpose Clinical Outcomes Assessments - Discussion Document for Patient-Focused Drug Development Public Workshop on Guidance 3: Select, Develop or Modify FitFor-Purpose Clinical Outcome Assessments, https://www. fda.gov/media/116277/download, Accessed October 23.

[13] Postuma RB, Berg D, Stern M, Poewe W, Olanow CW, Oertel W, Obeso J, Marek K, Litvan I, Lang AE, Halliday G, Goetz CG, Gasser T, Dubois B, Chan P, Bloem BR, Adler $\mathrm{CH}$, Deuschl G (2015) MDS clinical diagnostic criteria for Parkinson's disease. Mov Disord 30, 1591-1601.

[14] Patrick DL, Burke LB, Gwaltney CJ, Leidy NK, Martin ML, Molsen E, Ring L (2011) Content validity-establishing and reporting the evidence in newly developed patient-reported outcomes (PRO) instruments for medical product evaluation: ISPOR PRO good research practices task force report: Part 1-eliciting concepts for a new PRO instrument. Value Health 14, 967-977.
[15] Guest G, Bunce A, Johnson L (2006) How many interviews are enough? An experiment with data saturation and variability. Field Methods 18, 59-82.

[16] Turner-Bowker DM, Lamoureux RE, Stokes J, LitcherKelly L, Galipeau N, Yaworsky A, Solomon J, Shields AL (2018) Informing a priori sample size estimation in qualitative concept elicitation interview studies for clinical outcome assessment instrument development. Value Health 21, 839-842.

[17] (2001) ICH Harmonised Tripartite Guideline: Guideline for Good Clinical Practice. J Postgrad Med 47, 199-203.

[18] World Medical Association (2001) World Medical Association Declaration of Helsinki. Ethical principles for medical research involving human subjects. Bull World Health Organ 79, 373-374.

[19] U.S. Food and Drug Administration, Patient-focused drug development: Methods to identify what is important to patients; Guidance for industry, food and drug administration staff, and other stakeholders, https://www.fda.gov/ media/131230/download, Accessed July 23.

[20] Shoulson I, Longitudinal Examination of Problems that Bother People with Parkinson's, https://www.michaeljfox. org/grant/longitudinal-examination-problems-botherpeople-parkinsons, Accessed October 23.

[21] Vinikoor-Imler L, Arbatti L, Hosamath A, Sapir I, Shirvan J, Maserejian N, Shoulson I (2021) Cross-sectional profile of most bothersome problems as reported directly by individuals with Parkinson's disease (2697). Neurology 96, 2697.

[22] Humphrey L, Willgoss T, Trigg A, Meysner S, Kane M, Dickinson S, Kitchen H (2017) A comparison of three methods to generate a conceptual understanding of a disease based on the patients' perspective. J Patient Rep Outcomes 1,9 .

[23] Willgoss T, Cassater D, Connor S, Krishnan ML, Miller MT, Dias-Barbosa C, Phillips D, McCormack J, Bird LM, Burdine RD, Claridge S, Bichell TJ (2021) Measuring what matters to individuals with Angelman syndrome and their families: Development of a patient-centered disease concept model. Child Psychiatry Hum Dev 52, 654-668.

[24] Merino-Andreu M, Arnulf I, Konofal E, Derenne JP, Agid Y (2003) Unawareness of naps in Parkinson's disease and in disorders with excessive daytime sleepiness. Neurology 60, 1553-1554.

[25] Hurt CS, Rixon L, Chaudhuri KR, Moss-Morris R, Samuel M, Brown RG (2019) Barriers to reporting non-motor symptoms to health-care providers in people with Parkinson's. Parkinsonism Relat Disord 64, 220-225.

[26] Kandiah N, Narasimhalu K, Lau P-N, Seah S-H, Au WL, Tan LCS (2009) Cognitive decline in early Parkinson's disease. Mov Disord 24, 605-608.

[27] Kandiah N, Zhang A, Cenina AR, Au WL, Nadkarni N, Tan LC (2014) Montreal Cognitive Assessment for the screening and prediction of cognitive decline in early Parkinson's disease. Parkinsonism Relat Disord 20, 11451148.

[28] Jenkinson C, Fitzpatrick R, Peto V, Greenhall R, Hyman N (1997) The Parkinson's Disease Questionnaire (PDQ39): Development and validation of a Parkinson's disease summary index score. Age Ageing 26, 353-357.

[29] Goetz CG, Tilley BC, Shaftman SR, Stebbins GT, Fahn S, Martinez-Martin P, Poewe W, Sampaio C, Stern MB, Dodel R, Dubois B, Holloway R, Jankovic J, Kulisevsky J, Lang AE, Lees A, Leurgans S, LeWitt PA, Nyenhuis D, Olanow CW, Rascol O, Schrag A, Teresi JA, van Hilten JJ, LaPelle 
N (2008) Movement Disorder Society-sponsored revision of the Unified Parkinson's Disease Rating Scale (MDSUPDRS): Scale presentation and clinimetric testing results. Mov Disord 23, 2129-2170.

[30] Sarkar S, Raymick J, Imam S (2016) Neuroprotective and therapeutic strategies against Parkinson's disease: Recent perspectives. Int J Mol Sci 17, 904.
[31] Kalia LV, Lang AE (2015) Parkinson's disease. Lancet 386, 896-912.

[32] Tison F, Meissner WG (2013) Standard strategies for diagnosis and treatment of patients with newly diagnosed Parkinson disease: FRANCE. Neurology. Clin Pract 3, 480-481. 